\title{
A Hilbert transform for matrix functions on fractal domains
}

\author{
R. Abreu-Blaya*, J. Bory-Reyes ${ }^{\dagger}$, F. Brackx ${ }^{\ddagger}$, H. De Schepper ${ }^{\ddagger}$, F. Sommen ${ }^{\ddagger}$ \\ * Facultad de Informática y Matemática, Universidad de Holguín, Cuba \\ † Departamento de Matemática, Universidad de Oriente, Cuba \\ ‡ Clifford Research Group, Faculty of Engineering, Ghent University, Belgium
}

\begin{abstract}
We consider Hölder continuous circulant $(2 \times 2)$ matrix functions $\boldsymbol{G}_{2}^{1}$ defined on the fractal boundary $\Gamma$ of a Jordan domain $\Omega$ in $\mathbb{R}^{2 n}$. The main goal is to establish a Hilbert transform for such functions, within the framework of Hermitean Clifford analysis. This is a higher dimensional function theory centered around the simultaneous null solutions of two first order vector valued differential operators, called Hermitean Dirac operators. In [10] a Hermitean Cauchy integral was constructed by means of a matrix approach using circulant $(2 \times 2)$ matrix functions, from which a Hilbert transform was derived in [8], all of this for the case of domains with smooth boundary. However, crucial parts of the method used are not extendable to the case where the boundary of the considered domain is fractal. At present we propose an alternative approach which will enable us to define a new Hermitean Hilbert transform in that case. As a consequence, we are able to give necessary and sufficient conditions for the Hermitean monogenicity of a circulant matrix function $\mathbf{G}_{2}^{1}$ in the interior and exterior of $\Omega$, in terms of its boundary value $\mathbf{g}_{2}^{1}=\left.\mathbf{G}_{2}^{1}\right|_{\Gamma}$, extending in this way also results of [4] and [2], where $\Gamma$ is required to be Ahlfors-David regular.
\end{abstract}

Keywords: Hermitean Clifford analysis, Cauchy integral, Hilbert transform, fractal geometry.

Mathematics Subject Classification (2000): $30 \mathrm{G} 35$.

\section{Introduction}

In engineering sciences, and in particular in signal analysis, the one-dimensional Hilbert transform of a real signal $u(t)$, depending on a one-dimensional time variable $t$, has become a fundamental tool. For a suitable function or distribution $u(t)$ its Hilbert transform is given by the Cauchy Principal Value integral

$$
H[u](t)=-\frac{1}{\pi} \mathrm{Pv} \int_{-\infty}^{+\infty} \frac{u(\tau)}{\tau-t} d \tau
$$

Though initiated by Hilbert, the concept of a "conjugated pair" $(u, H[u])$, nowadays called a Hilbert pair, was developed mainly by Titchmarch and Hardy. 
The multidimensional approach to the Hilbert transform usually is a tensorial one, involving the so-called Riesz transforms in each of the cartesian variables separately. As opposed to this tensor approach, Clifford analysis (see e.g. [11, 18, 24, 23, 25]) is particularly suited for a treatment of multidimensional phenomena, encompassing all dimensions simultaneously as an intrinsic feature. Clifford analysis essentially is a higher dimensional function theory offering both a generalization of the theory of holomorphic functions in the complex plane and a refinement of classical multidimensional harmonic analysis. In the standard case, so-called Euclidean Clifford analysis focuses on monogenic functions, i.e. null solutions of the rotation invariant vector valued Dirac operator $\partial_{\underline{X}}=\sum_{j=1}^{m} e_{j} \partial_{x_{j}}$ where $\left(e_{1}, \ldots, e_{m}\right)$ is an orthonormal basis for the vector space $\mathbb{R}^{m}$ underlying the construction of the real Clifford algebra $\mathbb{R}_{0, m}$. The multidimensional Hilbert transform in the orthogonal Clifford framework and the related theory of Hardy spaces are nowadays well established, see [25, 17, 9]. However we want to draw the attention on the paper [27] of Horváth who, to our knowledge, was the first to define a vector valued Hilbert transform using Clifford algebra.

More recently Hermitean Clifford analysis has emerged as yet a refinement of the Euclidean setting, for the case of $\mathbb{R}^{2 n} \cong \mathbb{C}^{n}$. Here, Hermitean monogenic functions are considered, i.e. functions taking values in the complex Clifford algebra $\mathbb{C}_{2 n}$ and being simultaneous null solutions of two complex Hermitean Dirac operators, which are invariant under the action of the unitary group. The study of complex Dirac operators (also in other settings) was initiated in $[30,29,31]$; however, a systematic development of the Hermitean function theory is still in full progress, see e.g. $[15,6,7,14,12,2,4,5,3]$.

In [8] a new Hermitean Hilbert transform is introduced, arising naturally as a part of non-tangential boundary limit of a Hermitean Cauchy integral constructed in [10] for domains with $C^{\infty}$ smooth boundaries, using a matrix approach with circulant $(2 \times 2)$ matrix functions. More recently, see [2], the above operators were redefined in the context of Ahlfors-David surfaces and applied to solve boundary value problems for Hermitean monogenic matrix functions. The case of fractal boundaries, however, is not covered by the method developed there.

In this paper we introduce an alternative way of defining the Hermitean matrix Hilbert transform over a fractal surface which bounds a Jordan domain. We study its properties and apply them to solve boundary value problems for Hermitean monogenic matrix functions in fractal domains. As a consequence, also some results of [2] are extended to this more general context.

\section{Preliminaries}

\subsection{The Hermitean Clifford analysis setting}

Let $\left(e_{1}, \ldots, e_{m}\right)$ be an orthonormal basis of Euclidean space $\mathbb{R}^{m}$ and consider the complex Clifford algebra $\mathbb{C}_{m}$ constructed over $\mathbb{R}^{m}$. The non-commutative 
multiplication in $\mathbb{C}_{m}$ is governed by the rules:

$$
\begin{aligned}
e_{j}^{2} & =-1, & & j=1, \ldots, m \\
e_{j} e_{k}+e_{k} e_{j} & =0, & & j \neq k
\end{aligned}
$$

$\mathbb{C}_{m}$ is then generated additively by elements of the form $e_{A}=e_{j_{1}} \ldots e_{j_{k}}$, where $A=\left\{j_{1}, \ldots, j_{k}\right\} \subset\{1, \ldots, m\}$ with $j_{1}<\cdots<j_{k}$, while for $A=\emptyset$, one puts $e_{\emptyset}=1$, the identity element. Any Clifford number $\lambda \in \mathbb{C}_{m}$ may thus be written as $\lambda=\sum_{A} \lambda_{A} e_{A}, \lambda_{A} \in \mathbb{C}$, its Hermitean conjugate $\lambda^{\dagger}$ being defined by $\lambda^{\dagger}=\sum_{A} \lambda_{A}^{c} \bar{e}_{A}$, where the bar denotes the real Clifford algebra conjugation, i.e. the main anti-involution for which $\bar{e}_{j}=-e_{j}$, and $\lambda_{A}^{c}$ stands for the complex conjugate of the complex number $\lambda_{A}$. Euclidean space $\mathbb{R}^{m}$ is embedded in the Clifford algebra $\mathbb{C}_{m}$ by identifying $\left(x_{1}, \ldots, x_{m}\right)$ with the real Clifford vector $\underline{X}$ given by $\underline{X}=\sum_{j=1}^{m} e_{j} x_{j}$, for which $\underline{X}^{2}=-<\underline{X}, \underline{X}>=-|\underline{X}|^{2}$. The Fischer dual of $\underline{X}$ is the vector valued first order Dirac operator $\partial_{\underline{X}}=\sum_{j=1}^{m} e_{j} \partial_{x_{j}}$, factorizing the Laplacian: $\Delta_{m}=-\partial_{\underline{X}}^{2}$; it underlies the notion of monogenicity of a function, the higher dimensional counterpart of holomorphy in the complex plane. The considered functions are defined on (open subsets of) $\mathbb{R}^{m}$ and take values in the Clifford algebra $\mathbb{C}_{m}$. They are of the form $g=\sum_{A} g_{A} e_{A}$, with $g_{A}$ complex valued. Whenever a property such as continuity, differentiability, etc. is ascribed to $g$ it is meant that all components $g_{A}$ show that property. A Clifford algebra valued function $g$, defined and differentiable in an open region $\Omega$ of $\mathbb{R}^{m}$, is then called (left) monogenic in $\Omega$ iff $\partial_{\underline{X}} g=0$ in $\Omega$.

The transition from Euclidean Clifford analysis as described above to the Hermitean Clifford setting is essentially based on the introduction of a complex structure $J$, i.e. an $\mathrm{SO}(m)$ element, satisfying $J^{2}=-\mathbf{1}_{m}$. Since such an element can not exist when the dimension of the vector space is odd, we put $m=2 n$ from now on. In terms of the chosen orthonormal basis, a possible realization of the complex structure is $J\left[e_{2 j-1}\right]=-e_{2 j}$ and $J\left[e_{2 j}\right]=e_{2 j-1}, j=1, \ldots, n$. Two projection operators $\pm \frac{1}{2}\left(\mathbf{1}_{2 n} \pm i J\right)$ associated to $J$ then produce the main objects of Hermitean Clifford analysis by acting upon the corresponding objects in the Euclidean setting, see $[6,7]$. The vector space $\mathbb{C}^{2 n}$ thus decomposes as $W^{+} \oplus W^{-}$into two isotropic subspaces. The real Clifford vector is now denoted

$$
\underline{X}=\sum_{j=1}^{n}\left(e_{2 j-1} x_{2 j-1}+e_{2 j} x_{2 j}\right)
$$

with the corresponding Dirac operator

$$
\partial_{\underline{X}}=\sum_{j=1}^{n}\left(e_{2 j-1} \partial_{x_{2 j-1}}+e_{2 j} \partial_{x_{2 j}}\right)
$$

while we will also consider their so-called 'twisted' counterparts, obtained through the action of $J$, i.e.

$$
\begin{aligned}
\underline{X} \mid & =\sum_{j=1}^{n}\left(e_{2 j-1} x_{2 j}-e_{2 j} x_{2 j-1}\right) \\
\partial_{\underline{X}} & =\sum_{j=1}^{n}\left(e_{2 j-1} \partial_{x_{2 j}}-e_{2 j} \partial_{x_{2 j-1}}\right)
\end{aligned}
$$


As was the case with $\partial_{X}$, a notion of monogenicity may be associated in a natural way to $\partial_{\underline{X}}$ as well. The projections of the vector variable $\underline{X}$ on the spaces $W^{ \pm}$then yield the Hermitean Clifford variables $\underline{Z}$ and $\underline{Z}^{\dagger}$, given by

$$
\underline{Z}=\frac{1}{2}(\underline{X}+i \underline{X} \mid) \quad \text { and } \quad \underline{Z}^{\dagger}=-\frac{1}{2}(\underline{X}-i \underline{X} \mid)
$$

and those of the Dirac operator $\partial_{X}$ yield (up to a factor) the Hermitean Dirac operators $\partial_{\underline{Z}}$ and $\partial_{\underline{Z}^{\dagger}}$, given by

$$
\partial_{\underline{Z}}=-\frac{1}{4}\left(\partial_{\underline{X}}-i \partial_{\underline{X}}\right) \quad \text { and } \quad \partial_{\underline{Z}^{\dagger}}=\frac{1}{4}\left(\partial_{\underline{X}}+i \partial_{\underline{X}}\right)
$$

The Hermitean vector variables and Dirac operators are isotropic, i.e. $(\underline{Z})^{2}=$ $\left(\underline{Z}^{\dagger}\right)^{2}=0$ and $\left(\partial_{\underline{Z}}\right)^{2}=\left(\partial_{Z^{\dagger}}\right)^{2}=0$, whence the Laplacian allows for the decomposition $\Delta_{2 n}=4\left(\partial_{Z} \partial_{Z^{\dagger}}+\partial_{Z^{\dagger}} \partial_{Z}\right)$. These objects lie at the core of the Hermitean function theory by means of the following definition (see e.g. [6, 15]).

Definition 1 A continuously differentiable function $g$ in $\Omega \subset \mathbb{R}^{2 n}$ with values in $\mathbb{C}_{2 n}$ is called left Hermitean monogenic (or left h-monogenic) in $\Omega$, iff it satisfies the system $\partial_{\underline{Z}} g=0=\partial_{\underline{Z}^{\dagger}} g$ or the equivalent system $\partial_{\underline{X}} g=0=\partial_{\underline{X}} g$.

In a similar way right h-monogenicity is defined. Functions which are both left and right h-monogenic are called two-sided h-monogenic. The above definition inspires the statement that h-monogenicity constitutes a refinement of monogenicity, since h-monogenic functions (either left or right) are monogenic w.r.t. both Dirac operators $\partial_{\underline{X}}$ and $\partial_{\underline{X}}$.

\subsection{The transition to a circulant matrix approach}

The fundamental solutions of the Dirac operators $\partial_{\underline{X}}$ and $\partial_{\underline{X}}$ are respectively given by

$$
E(\underline{X})=-\frac{1}{a_{2 n}} \frac{\underline{X}}{|\underline{X}|^{2 n}}, \quad E \mid(\underline{X})=-\frac{1}{a_{2 n}} \frac{\underline{X} \mid}{|\underline{X}|^{2 n}}, \quad \underline{X} \in \mathbb{R}^{2 n} \backslash\{0\}
$$

where $a_{2 n}$ denotes the surface area of the unit sphere in $\mathbb{R}^{2 n}$. Introducing the functions

$$
\mathcal{E}(\underline{Z})=-(E+i E \mid)=\frac{2}{a_{2 n}} \frac{\underline{Z}}{|\underline{Z}|^{2 n}} \quad \text { and } \quad \mathcal{E}^{\dagger}(\underline{Z})=(E-i E \mid)=\frac{2}{a_{2 n}} \frac{\underline{Z}^{\dagger}}{|\underline{Z}|^{2 n}}
$$

these are not the fundamental solutions to the respective Hermitean Dirac operators $\partial_{\underline{Z}}$ and $\partial_{\underline{Z}^{\dagger}}$. However, introducing the particular circulant $(2 \times 2)$ matrices

$$
\mathcal{D}_{\left(\underline{Z}, \underline{Z}^{\dagger}\right)}=\left(\begin{array}{cc}
\partial_{\underline{Z}} & \partial_{\underline{Z}^{\dagger}} \\
\partial_{\underline{Z}^{\dagger}} & \partial_{\underline{Z}}
\end{array}\right), \quad \mathcal{E}=\left(\begin{array}{cc}
\mathcal{E} & \mathcal{E}^{\dagger} \\
\mathcal{E}^{\dagger} & \mathcal{E}
\end{array}\right) \quad \text { and } \quad \delta=\left(\begin{array}{cc}
\delta & 0 \\
0 & \delta
\end{array}\right),
$$

where $\delta$ is the Dirac delta distribution, one obtains that

$$
\mathcal{D}_{\left(\underline{Z}, \underline{Z}^{\dagger}\right)} \mathcal{E}(\underline{Z})=\boldsymbol{\delta}(\underline{Z})
$$


so that $\mathcal{E}$ may be considered as a fundamental solution of $\mathcal{D}_{\left(Z, Z^{\dagger}\right)}$ in a matricial context, see e.g. [8, 10, 29]. Moreover, the Dirac matrix $\mathcal{D}_{\left(\underline{Z}, \underline{Z}^{\dagger}\right)}$ in some sense factorizes the Laplacian, since

$$
4 \mathcal{D}_{\left(\underline{Z}, \underline{Z}^{\dagger}\right)}\left(\mathcal{D}_{\left(\underline{Z}, \underline{Z}^{\dagger}\right)}\right)^{\dagger}=\left(\begin{array}{cc}
\Delta_{2 n} & 0 \\
0 & \Delta_{2 n}
\end{array}\right) \equiv \boldsymbol{\Delta}
$$

where $\Delta_{2 n}$ is the usual Laplace operator in $\mathbb{R}^{2 n}$. This observation has lead to the idea of following a matrix approach in order to establish integral representation formulae in the Hermitean setting, see $[10,13]$. Moreover, it inspired the following definition.

Definition 2 Let $g_{1}, g_{2}$ be continuously differentiable functions defined in $\Omega$ and taking values in $\mathbb{C}_{2 n}$, and consider the matrix function

$$
\boldsymbol{G}_{2}^{1}=\left(\begin{array}{ll}
g_{1} & g_{2} \\
g_{2} & g_{1}
\end{array}\right)
$$

Then $\boldsymbol{G}_{2}^{1}$ is called left (respectively right) $\mathbf{H}$-monogenic in $\Omega$ if and only if it satisfies in $\Omega$ the system $\mathcal{D}_{\left(\underline{Z}, \underline{Z}^{\dagger}\right)} \boldsymbol{G}_{2}^{1}=\boldsymbol{O}$ (respectively $\left.\boldsymbol{G}_{2}^{1} \mathcal{D}_{\left(\underline{Z}, \underline{Z}^{\dagger}\right)}=\boldsymbol{O}\right)$. Here $\boldsymbol{O}$ denotes the matrix with zero entries.

Note that the $\mathbf{H}$-monogenicity of the matrix function $\boldsymbol{G}_{2}^{1}$ does not imply the h-monogenicity of its entry functions $g_{1}$ and $g_{2}$. However, choosing $g_{1}=g$ and $g_{2}=0$, the $\mathbf{H}$-monogenicity of the corresponding diagonal matrix, denoted $\boldsymbol{G}_{0}$, is equivalent to the h-monogenicity of the function $g$. Moreover, calling a matrix function $\boldsymbol{G}_{2}^{1}$ harmonic iff it satisfies the equation $\boldsymbol{\Delta}\left[\boldsymbol{G}_{2}^{1}\right]=\boldsymbol{O}$, each $\mathbf{H}$-monogenic matrix function $\boldsymbol{G}_{2}^{1}$ turns out to be harmonic, whence its entries are harmonic functions in the usual sense.

Notions of continuity, differentiability and integrability of $\boldsymbol{G}_{2}^{1}$ are introduced through the corresponding notions for its entries. In particular, we will need to define in this way the classes $\mathcal{C}^{0, \nu}(\mathbf{E})$ and $\boldsymbol{L}_{\boldsymbol{p}}(\mathbf{E})$ of, respectively, Hölder continuous and $p$-integrable circulant matrix functions over some suitable subset $\mathbf{E}$ of $\mathbb{R}^{2 n}$. However, introducing the non-negative function

$$
\left\|\boldsymbol{G}_{2}^{1}(\underline{X})\right\|=\max \left\{\left|g_{1}(\underline{X})\right|,\left|g_{2}(\underline{X})\right|\right\}
$$

these classes of circulant matrix functions may also be defined by means of the traditional conditions

$$
\left\|\boldsymbol{G}_{2}^{1}(\underline{X})-\boldsymbol{G}_{2}^{1}(\underline{Y})\right\| \leq c|\underline{X}-\underline{Y}|^{\nu}, \underline{X}, \underline{Y} \in \mathbf{E}
$$

and

$$
\int_{\mathbf{E}}\left\|\boldsymbol{G}_{2}^{1}(\underline{X})\right\|^{p}<+\infty
$$

respectively. From now on we denote by $c$ a generic positive constant, which can take different values.

\subsection{Box dimension and $d$-summable sets in $\mathbb{R}^{2 n}$}

Let $\mathbf{E}$ be an arbitrary subset of $\mathbb{R}^{2 n}$. Then for any $s \geq 0$ its Hausdorff measure $\mathcal{H}^{s}(\mathbf{E})$ may be defined by

$$
\mathcal{H}^{s}(\mathbf{E})=\lim _{\delta \rightarrow 0} \inf \left\{\sum_{k=1}^{\infty}\left(\operatorname{diam} B_{k}\right)^{s}: \mathbf{E} \subset \bigcup_{k=1}^{\infty} B_{k}, \operatorname{diam} B_{k}<\delta\right\}
$$


the infimum being taken over all countable $\delta$-coverings $\left\{B_{k}\right\}$ of $\mathbf{E}$ with open or closed balls. For $s=2 n$, the Hausdorff measure $\mathcal{H}^{2 n}$ coincides, up to a positive multiplicative constant, with the Lebesgue measure $\mathcal{L}^{2 n}$ in $\mathbb{R}^{2 n}$. Now, let $\mathbf{E}$ be compact. The Hausdorff dimension $\alpha_{H}(\mathbf{E})$ of $\mathbf{E}$ is then defined as the infimum of all $s \geq 0$ such that $\mathcal{H}^{s}(\mathbf{E})<+\infty$. For more details concerning the Hausdorff measure and dimension we refer to $[20,21]$. Frequently however, see [28], the so-called box dimension is used, defined for a compact set $\mathbf{E} \subset \mathbb{R}^{2 n}$ as

$$
\alpha(\mathbf{E})=\lim _{\varepsilon \rightarrow 0} \sup \frac{\log N_{\mathbf{E}}(\varepsilon)}{-\log \varepsilon}
$$

where $\mathbf{N}_{\mathbf{E}}(\varepsilon)$ stands for the minimal number of $\varepsilon$-balls needed to cover $\mathbf{E}$. Note that the limit above remains unchanged if $\mathbf{N}_{\mathbf{E}}(\varepsilon)$ is replaced by the number of $k$-cubes, with $2^{-k} \leq \varepsilon<2^{-k+1}$, intersecting $\mathbf{E}$. A cube $Q$ is called a $k$-cube if it is of the form $\left[l_{1} 2^{-k},\left(l_{1}+1\right) 2^{-k}\right] \times \cdots \times\left[l_{2 n} 2^{-k},\left(l_{2 n}+1\right) 2^{-k}\right]$, where $k$ and $l_{1}, \ldots, l_{2 n}$ are integers. The box dimension and the Hausdorff dimension of a given compact set $\mathbf{E}$ can be equal, which is for instance the case for the so-called $(2 n-1)$-rectifiable sets (see [22]), but in general we have that $\alpha_{H}(\mathbf{E}) \leq \alpha(\mathbf{E})$. The following geometric notion was in [26], and is essential in their method of integrating a form over a fractal boundary.

Definition 3 The compact set $\mathbf{E}$ is said to be d-summable iff the improper integral $\int_{0}^{1} N_{\mathbf{E}}(x) x^{d-1} d x$ converges.

\section{Lemma 1 It holds that}

(i) any d-summable set $\mathbf{E}$ has box dimension $\alpha(\mathbf{E}) \leq d$;

(ii) if $\alpha(\mathbf{E})<d$, then $\mathbf{E}$ is d-summable;

(iii) if $\mathbf{E}$ is $d$-summable, then it is also $(d+\varepsilon)$-summable for every $\varepsilon>0$.

In what follows, we will take $\Omega \subset \mathbb{R}^{2 n}$ to be a Jordan domain, i.e. a bounded oriented connected open subset of $\mathbb{R}^{2 n}$, the boundary $\Gamma$ of which is a compact topological surface. For our purpose, we will assume that the Hausdorff and box dimensions of $\Gamma$ satisfy $2 n-1 \leq \alpha_{H}(\Gamma) \leq \alpha(\Gamma)<2 n$. Note that this includes the case when $\Gamma$ is fractal in the sense of Mandelbrot, i.e. when $2 n-1<\alpha_{H}(\Gamma)$. Under these conditions, there will always exist $d \in[2 n-1,2 n[$ such that $\Gamma$ is $d$-summable, see Lemma 1 .

We will also need the so-called Whitney decomposition of $\Omega$, which we will only recall briefly; for details we refer to [32]. Consider the lattice $\mathbb{Z}^{2 n}$ in $\mathbb{R}^{2 n}$ and the collection of closed unit cubes defined by it; let $\mathcal{M}_{1}$ be the mesh consisting of those unit cubes having a non-empty intersection with $\Omega$. We then recursively define the meshes $\mathcal{M}_{k}, k=2,3, \ldots$, each time bisecting the sides of the cubes of the previous one. The cubes in $\mathcal{M}_{k}$ thus have side length $2^{-k+1}$ and diameter $|Q|=\sqrt{2 n} 2^{-k+1}$. We then define, for $k=2,3, \ldots$,

$$
\begin{aligned}
\mathcal{W}^{1}= & \left\{Q \in \mathcal{M}_{1} \mid \text { all neighbour cubes of } Q \text { belong to } \Omega\right\} \\
\mathcal{W}^{k}= & \left\{Q \in \mathcal{M}_{k} \mid \text { all neighbour cubes of } Q \text { belong to } \Omega,\right. \text { and } \\
& \left.\nexists Q^{*} \in \mathcal{W}^{k-1}: Q \subset Q^{*}\right\}
\end{aligned}
$$


for which it can be proven that

$$
\Omega=\bigcup_{k=1}^{+\infty} \mathcal{W}^{k}=\bigcup_{k=1}^{+\infty} \bigcup_{Q \in \mathcal{W}^{k}} Q \equiv \bigcup_{Q \in \mathcal{W}} Q
$$

all cubes $Q$ in the Whitney decomposition $\mathcal{W}$ of $\Omega$ having disjoint interiors. We then have the following relation between the $d$-summability of the boundary $\Gamma$ and the Whitney decomposition of $\Omega$.

Lemma 2 [26] If $\Omega$ is a Jordan domain of $\mathbb{R}^{2 n}$ and its boundary $\Gamma$ is $d$ summable, then the expression $\sum_{Q \in \mathcal{W}}|Q|^{d}$, called the d-sum of the Whitney decomposition $\mathcal{W}$ of $\Omega$, is finite.

\section{A Hermitean Hilbert transform on $d$-summable surfaces}

For further use, we introduce the notations $\Omega^{+} \equiv \Omega$, and $\Omega^{-} \equiv \mathbb{R}^{2 n} \backslash \bar{\Omega}$. From now on we reserve the notations $\underline{Y}$ and $\underline{Y} \mid$ for Clifford vectors associated to points in $\Omega^{ \pm}$, while their Hermitean counterparts are denoted by $\underline{V}=\frac{1}{2}(\underline{Y}+i \underline{Y} \mid)$ and $\underline{V}^{\dagger}=-\frac{1}{2}(\underline{Y}-i \underline{Y} \mid)$. By means of the above matrix approach, the following Hermitean Borel-Pompeiu formula was established in [10], however for the case of a domain with smooth boundary.

Theorem 1 Let $\Omega$ be a Jordan domain in $\mathbb{R}^{2 n}$ with piecewise $C^{\infty}$ smooth boundary $\Gamma$, and let $\boldsymbol{G}_{2}^{1} \in \mathcal{C}\left(\Omega ; \mathbb{C}_{2 n}\right)$. It then holds that

$$
\mathcal{C}_{\Gamma} \boldsymbol{G}_{2}^{1}(\underline{Y})+\mathcal{T}_{\Omega} \mathcal{D}_{\left(\underline{Z}, \underline{Z}^{\dagger}\right)} \boldsymbol{G}_{2}^{1}(\underline{Y})= \begin{cases}(-1)^{\frac{n(n+1)}{2}}(2 i)^{n} \boldsymbol{G}_{2}^{1}(\underline{Y}), & \underline{Y} \in \Omega^{+} \\ 0, & \underline{Y} \in \Omega^{-}\end{cases}
$$

where $\mathcal{C}_{\Gamma} \boldsymbol{G}_{2}^{1}$ is the Hermitean Cauchy integral, given by

$$
\mathcal{C}_{\Gamma} \boldsymbol{G}_{2}^{1}(\underline{Y})=\int_{\Gamma} \mathcal{E}(\underline{Z}-\underline{V}) \mathbf{N}_{\left(\underline{Z}, \underline{Z}^{\dagger}\right)} \boldsymbol{G}_{2}^{1}(\underline{X}) d \mathcal{H}^{2 n-1}, \underline{Y} \in \Omega^{ \pm}
$$

The circulant matrix

$$
\mathbf{N}_{\left(\underline{Z}, \underline{Z}^{\dagger}\right)}=\left(\begin{array}{cc}
\underline{N} & -\underline{N}^{\dagger} \\
-\underline{N}^{\dagger} & \underline{N}
\end{array}\right)
$$

contains (up to a constant factor) the Hermitean projections $\underline{N}$ and $\underline{N}^{\dagger}$ of the unit normal vector $\underline{n}(\underline{X})$ at the point $\underline{X} \in \Gamma$. Furthermore, $\mathcal{T}_{\Omega}$ denotes the Hermitean Téodorescu transform, given for $\boldsymbol{F}_{2}^{1} \in \mathcal{C}^{1}(\Omega)$ by

$$
\mathcal{T}_{\Omega} \boldsymbol{F}_{2}^{1}(\underline{Y})=-\int_{\Omega} \mathcal{E}(\underline{Z}-\underline{V}) \boldsymbol{F}_{2}^{1}(\underline{X}) d W\left(\underline{Z}, \underline{Z}^{\dagger}\right)
$$

where $d W\left(\underline{Z}, \underline{Z}^{\dagger}\right)$ is the associated volume element, given by

$$
d V(\underline{X})=(-1)^{\frac{n(n-1)}{2}}\left(\frac{i}{2}\right)^{n} d W\left(\underline{Z}, \underline{Z}^{\dagger}\right)
$$

reflecting integration in the respective underlying complex planes. 
Based on the above definition of the Hermitean Cauchy integral, which is in fact defined for any $\boldsymbol{G}_{2}^{1} \in \boldsymbol{L}_{\mathbf{2}}(\Gamma)$, a matricial Hermitean Hilbert transform was introduced in [8], by taking non-tangential boundary limits.

Theorem 2 Let $\Omega$ be a Jordan domain in $\mathbb{R}^{2 n}$ with piecewise $C^{\infty}$ smooth boundary $\Gamma$, and let $\boldsymbol{G}_{2}^{1} \in \boldsymbol{L}_{\mathbf{2}}(\Gamma)$. Then the non-tangential boundary values of the Hermitean Cauchy integral $\mathcal{C}_{\Gamma} \boldsymbol{G}_{2}^{1}$ are given by

$$
\lim _{\substack{\underline{Y} \rightarrow \underline{U} \\ \underline{\underline{Y}} \in \Omega^{ \pm}}} \mathcal{C}\left[\boldsymbol{G}_{2}^{1}\right](\underline{Y})=(-1)^{\frac{n(n+1)}{2}}(2 i)^{n}\left( \pm \frac{1}{2} \boldsymbol{G}_{2}^{1}(\underline{U})+\frac{1}{2} \mathcal{H}\left[\boldsymbol{G}_{2}^{1}\right](\underline{U})\right), \quad \underline{U} \in \Gamma
$$

which take the form of the usual Plemelj-Sokhotski formulae and where $\mathcal{H}$ is the matrix operator

$$
\mathcal{H}=\frac{1}{2}\left(\begin{array}{rr}
H+H \mid & -H+H \mid \\
-H+H \mid & H+H \mid
\end{array}\right)
$$

defined in terms of the Clifford-Hilbert operator $H$ and its twisted analogue $H \mid$.

It was also shown that $\mathcal{H}$ has all usual properties of a Hilbert operator.

In [1], the result of Theorem 1 was extended to the case of domains with $d$-summable surface, by means of the following definition.

Definition 4 Let $\Omega$ be a Jordan domain in $\mathbb{R}^{2 n}$ with d-summable boundary $\Gamma$, with $d \in\left[2 n-1,2 n\left[\right.\right.$. Let moreover $d-2 n+1<\nu \leq 1$ and consider $\boldsymbol{G}_{2}^{1} \in \mathcal{C}^{0, \nu}(\Gamma)$. Then, for $\underline{Y} \in \mathbb{R}^{2 n} \backslash \Gamma$ the Hermitean Cauchy integral of $\boldsymbol{G}_{2}^{1}$ is defined by

$$
\left(\mathcal{C}_{\Gamma}^{*} \boldsymbol{G}_{2}^{1}\right)(\underline{Y})=(-1)^{\frac{n(n+1)}{2}}(2 i)^{n} \boldsymbol{\chi}_{\Omega}(\underline{Y}) \widetilde{\boldsymbol{G}}_{2}^{1}(\underline{Y})-\boldsymbol{\mathcal { T }}_{\Omega} \mathcal{D}_{\left(\underline{Z}, \underline{Z}^{\dagger}\right)} \widetilde{\boldsymbol{G}}_{2}^{1}(\underline{Y})
$$

$\chi_{\Omega}$ being the diagonal matrix version of the associated standard characteristic function $\chi_{\Omega}$ of $\Omega$.

Here, $\widetilde{\boldsymbol{G}}_{2}^{1}$ denotes a Whitney type extension of $\boldsymbol{G}_{2}^{1}$, as meant in the extension theorem below, see [32].

Theorem 3 (Whitney Extension Theorem) Let $\mathbf{E} \subset \mathbb{R}^{2 n}$ be compact and $\boldsymbol{G}_{2}^{1} \in \mathcal{C}^{0, \nu}(\mathbf{E})$. Then, there exists a compactly supported matrix function $\widetilde{\boldsymbol{G}}_{2}^{1}$ satisfying

(i) $\left.\widetilde{\boldsymbol{G}}_{2}^{1}\right|_{\mathbf{E}}=\boldsymbol{G}_{2}^{1}$;

(ii) $\widetilde{\boldsymbol{G}}_{2}^{1} \in \mathcal{C}^{\infty}\left(\mathbb{R}^{2 n} \backslash \mathbf{E}\right)$;

(iii) $\left\|\mathcal{D}_{\left(\underline{Z}, \underline{Z}^{\dagger}\right)} \widetilde{\boldsymbol{G}}_{2}^{1}(\underline{X})\right\| \leq c \operatorname{dist}(\underline{X}, \mathbf{E})^{\nu-1}$, for $\underline{X} \in \mathbb{R}^{2 n} \backslash \mathbf{E}$.

Direct verification shows that $\mathcal{C}_{\Gamma}^{*} \boldsymbol{G}_{2}^{1}$, being Hermitean monogenic in $R^{2 n} \backslash \Gamma$, vanishes at infinity, while, in cases where $\Gamma$ is sufficiently regular (e.g. AhlforsDavid regular), the Hermitean Cauchy integral (1) reduces to the one considered in [2]. Furthermore, Definition 4 is legitimate, since the right hand side of (1) exists for any $\underline{Y} \in \mathbb{R}^{2 n} \backslash \Gamma$ and does not depend on the particular choice of the Whitney type extension $\widetilde{\boldsymbol{G}}_{2}^{1}$. A proof of this last assertion can be found in [1]. 
A natural question is whether $\mathcal{C}_{\Gamma}^{*} \boldsymbol{G}_{2}^{1}$ admits a continuous extension to $\bar{\Omega}=$ $\Omega \cup \Gamma$, in which case, following the traditional structure of the Plemelj-Sokhotski formulae, a "fractal" Hermitean Hilbert transform could be introduced as follows:

$$
\mathcal{H}_{\Gamma}^{*} \boldsymbol{G}_{2}^{1}(\underline{X})=2 \frac{(-1)^{\frac{n(n+1)}{2}}}{(2 i)^{n}}\left(\mathcal{C}_{\Gamma}^{*} \boldsymbol{G}_{2}^{1}\right)^{+}(\underline{X})-\boldsymbol{G}_{2}^{1}(\underline{X}), \quad \underline{X} \in \Gamma
$$

$\left(\mathcal{C}_{\Gamma}^{*} \boldsymbol{G}_{2}^{1}\right)^{+}$denoting the trace on $\Gamma$ of the continuous extension of $\mathcal{C}_{\Gamma}^{*} \boldsymbol{G}_{2}^{1}$ to $\bar{\Omega}$. Definition (2) would then provide an alternative for the matricial Hermitean Hilbert transform $\mathcal{H}$ introduced in [8] for domains with $C^{\infty}$ smooth boundaries, see Theorem 2 above. Now, under an additional condition on the regularity of the considered matrix function $\boldsymbol{G}_{2}^{1}$, this question indeed has an affirmative answer, as stated in the following theorem.

Theorem 4 Let $\Omega$ be a Jordan domain in $\mathbb{R}^{2 n}$ with d-summable boundary $\Gamma$, with $d \in[2 n-1,2 n[$. Furthermore, let $d-2 n+1<\nu \leq 1$ and consider $\boldsymbol{G}_{2}^{1} \in \mathcal{C}^{0, \nu}(\Gamma)$. If moreover

$$
\nu>\frac{d}{2 n}
$$

then $\mathcal{C}_{\Gamma}^{*} \boldsymbol{G}_{2}^{1}(\underline{X})$ admits a continuous extension to $\bar{\Omega}$. Moreover, it then holds that the Hilbert transform $\mathcal{H}_{\Gamma}^{*} \boldsymbol{G}_{2}^{1}$, defined by (2), belongs to $\mathcal{C}^{0, \mu}(\Gamma)$, whenever

$$
\mu<\frac{2 n \nu-d}{2 n-d}
$$

Proof.

Since $\nu>\frac{d}{2 n}$ implies that $2 n<\frac{2 n-d}{1-\nu}$, we may choose $p$ such that $2 n<p<\frac{2 n-d}{1-\nu}$. We will now first show, for any such $p$, that $\mathcal{D}_{\left(\underline{Z}, \underline{Z}^{\dagger}\right)} \widetilde{\boldsymbol{G}}_{2}^{1} \in \boldsymbol{L}_{\boldsymbol{p}}(\Omega)$. To this end, let $\mathcal{W}=\bigcup_{k=1}^{\infty} \mathcal{W}^{k}$ be the Whitney decomposition of $\Omega$. Then we have

$$
\begin{aligned}
\int_{\Omega}\left\|\mathcal{D}_{\left(\underline{Z}, \underline{Z}^{\dagger}\right)} \widetilde{\boldsymbol{G}}_{2}^{1}(\underline{Y})\right\|^{p} & =\sum_{Q \in \mathcal{W}} \int_{\Omega}\left\|\mathcal{D}_{\left(\underline{Z}, \underline{Z}^{\dagger}\right)} \widetilde{\boldsymbol{G}}_{2}^{1}(\underline{Y})\right\|^{p} \\
& \leq c \sum_{Q \in \mathcal{W}} \int_{Q} \operatorname{dist}(\underline{Y}, \Gamma)^{-p(1-\nu)} d V(\underline{Y})
\end{aligned}
$$

the last inequality following from Theorem 3(iii). By construction of the Whitney decomposition of $\Omega$, we have that, for any $Q \in \mathcal{W}$,

$$
\operatorname{dist}(\underline{Y}, \Gamma) \geq \frac{|Q|}{\sqrt{2 n}}, \quad \forall \underline{Y} \in Q
$$

see also [32], whence

$$
\int_{\Omega}\left\|\mathcal{D}_{\left(\underline{Z}, \underline{Z}^{\dagger}\right)} \widetilde{\boldsymbol{G}}_{2}^{1}(\underline{Y})\right\|^{p} \leq c \sum_{Q \in \mathcal{W}}|Q|^{2 n-p(1-\nu)}
$$

The finiteness of the last sum follows, on account of Lemma 2 , from the $d$ summability of $\Gamma$, together with the fact that $2 n-p(1-\nu)>d$. Hence we indeed have that $\mathcal{D}_{\left(\underline{Z}, \underline{Z}^{\dagger}\right)} \widetilde{\boldsymbol{G}}_{2}^{1} \in \boldsymbol{L}_{\boldsymbol{p}}(\Omega)$, so that the integral term in (1), viz

$$
\Phi(\underline{\mathbf{Y}})=\mathcal{T}_{\boldsymbol{\Omega}} \mathcal{D}_{\left(\underline{\mathbf{z}}, \underline{\mathbf{Z}}^{\dagger}\right)} \widetilde{G}_{\mathbf{2}}^{\mathbf{1}}(\underline{\mathbf{Y}})
$$


represents a continuous function in $\mathbb{R}^{2 n}$, see e.g. [23]. This clearly forces $\mathcal{C}_{\Gamma}^{*} \boldsymbol{G}_{2}^{1}(\underline{X})$ to admit a continuous extension to $\bar{\Omega}$, whence $\mathcal{H}_{\Gamma}^{*} \boldsymbol{G}_{2}^{1}$ is well defined. Moreover, $\mathbf{\Phi} \in \mathcal{C}^{\mathbf{0}, \frac{\mathbf{p}-\mathbf{2 n}}{\mathbf{p}}}\left(\mathbb{R}^{\mathbf{2 n}}\right)$, which implies that $\mathcal{H}_{\Gamma}^{*} \boldsymbol{G}_{2}^{1} \in \mathcal{C}^{0, \mu}(\Gamma)$ for any $\mu$ satisfying $\mu<\frac{2 n \nu-d}{2 n-d}$.

Remark 1 Observe that, still under condition (3), $\mathcal{H}_{\Gamma}^{*} \boldsymbol{G}_{2}^{1}$ may be rewritten as

$$
\mathcal{H}_{\Gamma}^{*} \boldsymbol{G}_{2}^{1}(\underline{X})=\boldsymbol{G}_{2}^{1}(\underline{X})-2 \frac{(-1)^{\frac{n(n+1)}{2}}}{(2 i)^{n}} \mathcal{T}_{\Omega} \mathcal{D}_{\left(\underline{Z}, \underline{Z}^{\dagger}\right)} \widetilde{\boldsymbol{G}}_{2}^{1}(\underline{X})
$$

on account of Theorem 1.

Remark 2 Similar results may be obtained for the case of right-hand versions of the above Hermitean Cauchy integrals and Hilbert transforms. To that end we only need to introduce the alternative definitions

$$
\left(\boldsymbol{G}_{2}^{1} \mathcal{H}_{\Gamma}^{*}\right)(\underline{X})=\boldsymbol{G}_{2}^{1}(\underline{X})-2 \frac{(-1)^{\frac{n(n+1)}{2}}}{(2 i)^{n}}\left(\widetilde{\boldsymbol{G}}_{2}^{1} \mathcal{D}_{\left(\underline{Z}, \underline{Z}^{\dagger}\right)} \boldsymbol{\mathcal { T }}_{\Omega}\right)(\underline{X})
$$

and

$$
\left(\boldsymbol{G}_{2}^{1} \mathcal{C}_{\Gamma}^{*}\right)(\underline{Y})=(-1)^{\frac{n(n+1)}{2}}(2 i)^{n} \boldsymbol{\chi}_{\Omega}(\underline{Y}) \widetilde{\boldsymbol{G}}_{2}^{1}(\underline{Y})-\left(\widetilde{\boldsymbol{G}}_{2}^{1} \mathcal{D}_{\left(\underline{Z}, \underline{Z}^{\dagger}\right.} \boldsymbol{\mathcal { T }}_{\Omega}\right)(\underline{Y})
$$

where for a matrix function $\boldsymbol{F}_{2}^{1}$

$$
\left(\boldsymbol{F}_{2}^{1} \mathcal{T}_{\Omega}\right)(\underline{Y})=-\int_{\Omega} \boldsymbol{F}_{2}^{1}(\underline{X}) \mathcal{E}(\underline{Z}-\underline{V}) d W\left(\underline{Z}, \underline{Z}^{\dagger}\right)
$$

\section{Boundary value theory}

In what follows, we will assume $\Gamma$ to be $d$-summable with $2 n-1 \leq d<2 n$ and we will take $\nu \in] d-2 n+1,1]$ such that condition (3) is fulfilled.

\subsection{Criteria for Hermitean monogenicity}

Theorem 5 Let $\boldsymbol{G}_{2}^{1} \in \mathcal{C}^{0, \nu}(\bar{\Omega})$ with trace $\boldsymbol{g}_{2}^{1}=\left.\boldsymbol{G}_{2}^{1}\right|_{\Gamma}$. Then the following statements are equivalent:

(i) $\boldsymbol{G}_{2}^{1}$ is $\mathbf{H}$-monogenic in $\Omega$;

(ii) $\boldsymbol{G}_{2}^{1}$ is harmonic in $\Omega$ and $\mathcal{H}_{\Gamma}^{*} \boldsymbol{g}_{2}^{1}=\boldsymbol{g}_{2}^{1}$.

Proof.

Assume $\boldsymbol{G}_{2}^{1}$ to be $\mathbf{H}$-monogenic in $\Omega$, then it also is harmonic in $\Omega$. Now, let $\widetilde{\boldsymbol{G}}_{2}^{1}$ be a Whitney type extension of $\boldsymbol{G}_{2}^{1} \in \mathcal{C}^{0, \nu}(\bar{\Omega})$. Since $\mathcal{D}_{\left(\underline{Z}, \underline{Z}^{\dagger}\right)} \boldsymbol{G}_{2}^{1}=\boldsymbol{O}$, we have that $\widetilde{\boldsymbol{G}}_{2}^{1}$ also is a Whitney type extension of $\boldsymbol{g}_{2}^{1}=\left.\boldsymbol{G}_{2}^{1}\right|_{\Gamma}$, whence we may use it when applying Definition 4 for $\mathcal{C}_{\Gamma}^{*} \boldsymbol{g}_{2}^{1}$. We obtain

$$
\mathcal{C}_{\Gamma}^{*} \boldsymbol{g}_{2}^{1}(\underline{Y})=(-1)^{\frac{n(n+1)}{2}}(2 i)^{n} \boldsymbol{G}_{2}^{1}(\underline{Y}), \quad \underline{Y} \in \Omega
$$


Next, we conclude from (4) that

$$
\left(\mathcal{C}_{\Gamma}^{*} \boldsymbol{g}_{2}^{1}\right)^{+}(\underline{X})=(-1)^{\frac{n(n+1)}{2}}(2 i)^{n} \boldsymbol{g}_{2}^{1}(\underline{X})
$$

from which we eventually obtain that $\boldsymbol{\mathcal { H }}_{\Gamma}^{*} \boldsymbol{g}_{2}^{1}=\boldsymbol{g}_{2}^{1}$. Conversely, assume (ii) to hold, and define

$$
\boldsymbol{\Psi}= \begin{cases}\mathcal{C}_{\Gamma}^{*} \boldsymbol{g}_{2}^{1}(\underline{Y}), & \underline{Y} \in \Omega, \\ (-1)^{\frac{n(n+1)}{2}}(2 i)^{n} \boldsymbol{g}_{2}^{1}(\underline{X}), & \underline{X} \in \Gamma\end{cases}
$$

Clearly the function $\boldsymbol{\Psi}$ is $\mathbf{H}$-monogenic, and hence harmonic in $\Omega$. For $\underline{X} \in \Gamma$, it follows that

$$
\left(\mathcal{C}_{\Gamma}^{*} \boldsymbol{g}_{2}^{1}\right)^{+}(\underline{X})=(-1)^{\frac{n(n+1)}{2}}(2 i)^{n} \boldsymbol{g}_{2}^{1}(\underline{X})
$$

since $\mathcal{H}_{\Gamma}^{*} \boldsymbol{g}_{2}^{1}=\boldsymbol{g}_{2}^{1}$. Therefore, $\boldsymbol{\Psi}$ also is continuous on $\bar{\Omega}$. On the other hand, as $\boldsymbol{G}_{2}^{1}-\Psi$ is harmonic in $\Omega$ and $\left.\left(\boldsymbol{G}_{2}^{1}-\Psi\right)\right|_{\Gamma}=0$, we have that $\boldsymbol{G}_{2}^{1}(\underline{Y})=\mathcal{C}_{\Gamma}^{*} \boldsymbol{g}_{2}^{1}(\underline{Y})$ for $\underline{Y} \in \Omega$. This clearly forces $\boldsymbol{G}_{2}^{1}$ to be $\mathbf{H}$-monogenic in $\Omega$.

Remark 3 Observe that the above formula (4) constitutes a small improvement of [1, Theorem 4], where it is a priori assumed that $\boldsymbol{G}_{2}^{1} \in \mathcal{C}^{1}(\bar{\Omega})$.

For right $\mathbf{H}$-monogenic functions the following analogue is obtained.

Theorem 6 Let $\boldsymbol{G}_{2}^{1} \in \mathcal{C}^{0, \nu}(\bar{\Omega})$ with trace $\boldsymbol{g}_{2}^{1}=\left.\boldsymbol{G}_{2}^{1}\right|_{\Gamma}$. Then the following statements are equivalent:

(i) $\boldsymbol{G}_{2}^{1}$ is right $\mathbf{H}$-monogenic in $\Omega$;

(ii) $\boldsymbol{G}_{2}^{1}$ is harmonic in $\Omega$ and $\boldsymbol{g}_{2}^{1} \mathcal{H}_{\Gamma}^{*}=\boldsymbol{g}_{2}^{1}$.

\subsection{A conservation law for two-sided H-monogenic func- tions}

This subsection is devoted to establishing a connection between the two-sided $\mathbf{H}$ monogenicity of a matrix function $\boldsymbol{G}_{2}^{1}$ in $\Omega$ and the Hermitean Hilbert transforms of its trace on $\Gamma$. The result established here extends the so-called "conservation law" obtained in [2] to the present more general context of fractal domains.

Theorem $\mathbf{7}$ Let $\boldsymbol{G}_{2}^{1} \in \mathcal{C}^{0, \nu}(\bar{\Omega})$ with trace $\boldsymbol{g}_{2}^{1}=\left.\boldsymbol{G}_{2}^{1}\right|_{\Gamma}$ be left $\mathbf{H}$-monogenic in $\Omega$. Then the following statements are equivalent:

(i) $\boldsymbol{G}_{2}^{1}$ is two-sided $\mathbf{H}$-monogenic in $\Omega$;

(ii) $\boldsymbol{H}_{\Gamma}^{*} \boldsymbol{g}_{2}^{1}=\boldsymbol{g}_{2}^{1} \mathcal{H}_{\Gamma}^{*}$.

Proof.

Assume that $\boldsymbol{G}_{2}^{1}$ is two-sided $\mathbf{H}$-monogenic in $\Omega$. Then, a combination of the Theorems 5 and 6 yields

$$
\mathcal{H}_{\Gamma}^{*} \boldsymbol{g}_{2}^{1}=\boldsymbol{g}_{2}^{1}=\boldsymbol{g}_{2}^{1} \mathcal{H}_{\Gamma}^{*}
$$

Conversely, assume that $\mathcal{H}_{\Gamma}^{*} \boldsymbol{g}_{2}^{1}=\boldsymbol{g}_{2}^{1} \mathcal{H}_{\Gamma}^{*}$. From the assumed left $\mathbf{H}$-monogenicity of $\boldsymbol{G}_{2}^{1}$, we have on account of (4), that

$$
(-1)^{\frac{n(n+1)}{2}}(2 i)^{n} \boldsymbol{G}_{2}^{1}=\mathcal{C}_{\Gamma}^{*} \boldsymbol{g}_{2}^{1}, \quad \text { in } \Omega
$$


Consequently, for $\underline{X} \in \Gamma$ we have

$$
\begin{aligned}
(-1)^{\frac{n(n+1)}{2}}(2 i)^{n} \boldsymbol{g}_{2}^{1}(\underline{X}) & =\left(\mathcal{C}_{\Gamma}^{*} \boldsymbol{g}_{2}^{1}\right)^{+}(\underline{X}) \\
& =\frac{1}{2}(-1)^{\frac{n(n+1)}{2}}(2 i)^{n}\left(\boldsymbol{g}_{2}^{1}(\underline{X})+\mathcal{H}_{\Gamma}^{*} \boldsymbol{g}_{2}^{1}(\underline{X})\right)
\end{aligned}
$$

or, equivalently, $\boldsymbol{g}_{2}^{1}(\underline{X})=\mathcal{H}_{\Gamma}^{*} \boldsymbol{g}_{2}^{1}(\underline{X})$. In view of the assumption made, we thus obtain that $\boldsymbol{g}_{2}^{1}(\underline{X})=\boldsymbol{g}_{2}^{1} \mathcal{H}_{\Gamma}^{*}(\underline{X})$. The two-sided $\mathbf{H}$-monogenicity of $\boldsymbol{G}_{2}^{1}$ in $\Omega$ is now implied by Theorem 6 .

\section{Acknowledgments}

This paper was written during a scientific stay of the first author at the Clifford Research Group of the Department of Mathematical Analysis of Ghent University, supported by a "Visiting Postdoctoral Fellowship" of the Flemish Research Foundation. He wishes to thank the members of the Clifford Research Group for their kind hospitality during this stay.

\section{References}

[1] R. Abreu Blaya, J. Bory Reyes, F. Brackx, H. De Schepper, F. Sommen, A Hermitean Cauchy formula on a domain with fractal boundary, submitted.

[2] R. Abreu Blaya et al., Hermitean Cauchy integral decomposition of continuous functions on hypersurfaces, Bound. Value Probl. vol. 2008 (2008), Article ID 425256, 16 pages.

[3] R. Abreu Blaya, J. Bory Reyes, F. Brackx, H. De Schepper, Hermitean Téodorescu transform decomposition of continuous matrix functions on fractal hypersurfaces, submitted.

[4] R. Abreu Blaya, J. Bory Reyes, D. Peña Peña, F. Sommen, A boundary value problem for Hermitian monogenic functions, Bound. Value Probl. vol. 2008 (2008), Article ID 385874, 7 pages.

[5] R. Abreu Blaya, J. Bory Reyes, T. Moreno García, Hermitian decomposition of continuous functions on a fractal surface, Bull. Braz. Math. Soc. 40(1) (2009), 107-115.

[6] F. Brackx et al., Fundaments of Hermitean Clifford analysis - Part I: Complex structure, Complex Anal. Oper. Theory 1(3) (2007), 341-365.

[7] F. Brackx et al., Fundaments of Hermitean Clifford analysis - Part II: Splitting of h-monogenic equations, Complex Var. Elliptic Equ. 52(1011) (2007), 1063-1079.

[8] F. Brackx, B. De Knock, H. De Schepper, A matrix Hilbert transform in Hermitean Clifford analysis, J. Math. Anal. Appl. 344 (2008), 1068-1078.

[9] F. Brackx, B. De Knock, H. De Schepper, D. Eelbode, On the interplay between the Hilbert transform and conjugate harmonic functions, Mathematical Methods in the Applied Sciences 29(12) (2006), 1435-1450. 
[10] F. Brackx, B. De Knock, H. De Schepper, F. Sommen, On Cauchy and Martinelli-Bochner integral formulae in Hermitean Clifford analysis, Bull Braz Math Soc. 40(3) (2009), 395-416.

[11] F. Brackx, R. Delanghe, F. Sommen, Clifford analysis, Research Notes in Mathematics 76, Pitman (Advanced Publishing Program), Boston, 1982.

[12] F. Brackx, H. De Schepper, D. Eelbode and V. Souček, The Howe dual pair in Hermitean Clifford analysis, to appear in Rev. Mat. Iberoamericana.

[13] F. Brackx, H. De Schepper, M.E. Luna Elizarrars, M. Shapiro, Integral representation formulae in Hermitean Clifford analysis. In: K. Gürlebeck and C. Könke (eds.), Proceedings of the 18th International Conference on the Application of Computer Science and Mathematics in Architecture and Civil Engineering, Weimar, Germany, 2009.

[14] F. Brackx, H. De Schepper, F. Sommen, A theoretical framework for wavelet analysis in a Hermitean Clifford setting, Comm. Pure Appl. Anal. 6(3), 2007, 549-567.

[15] F. Brackx, H. De Schepper, F. Sommen, The Hermitean Clifford analysis toolbox, Adv. Appl. Cliff. Alg. 18(3-4) (2008), 451-487.

[16] F. Colombo, I. Sabadini, F. Sommen, D.C. Struppa, Analysis of Dirac Systems and Computational Algebra, Birkhäuser, Boston, 2004.

[17] R. Delanghe, On Some Properties of the Hilbert Transform in Euclidean Space, Bull. Belg. Math. Soc. - Simon Stevin 11 (2004), 163-180.

[18] R. Delanghe, F. Sommen, V. Souček, Clifford Algebra and Spinor-Valued Functions, Kluwer Academic Publishers, Dordrecht, 1992.

[19] D. Eelbode, Stirling numbers and spin-Euler polynomials, Exp. Math. 16(1) (2007), 55-66.

[20] K.J. Falconer, The geometry of fractal sets, Cambridge Tracts in Mathematics 85, Cambridge University Press, Cambridge, 1986.

[21] J. Feder, Fractals (With a foreword by Benoit B. Mandelbrot), Physics of Solids and Liquids, Plenum Press, New York, 1988.

[22] H. Federer, Geometric measure theory, Die Grundlehren der mathematischen Wissenschaften 153, Springer Verlag, New York Inc., New York, 1969.

[23] K. Gürlebeck, K. Habetha, W. Sprössig, Holomorphic functions in the plane and $n$-dimensional space, translated from the 2006 German original, Birkhäuser Verlag, Basel, 2008.

[24] K. Gürlebeck, W. Sprössig, Quaternionic and Clifford Calculus for Physicists and Engineers, Wiley, Chichester, 1998.

[25] J. Gilbert, M. Murray, Clifford Algebras and Dirac Operators in Harmonic Analysis, Cambridge University Press, Cambridge, 1991. 
[26] J. Harrison, A. Norton, The Gauss-Green theorem for fractal boundaries, Duke Mathematical Journal 67(3) (1992), 575-588.

[27] J. Horváth, Sur les fonctions conjuguées à plusieurs variables (French), Koninklijke Nederlandse Akademie van Wetenschappen, Proceedings Series A $\mathbf{5 6}=$ Indagationes Mathematicae 15 (1953), 17-29.

[28] M.L. Lapidus, H. Maier, Hypothse de Riemann, cordes fractales vibrantes et conjecture de Weyl-Berry modifie (French), C.R. Acad. Sci. Paris Série I Math. 313(1) (1991), 19-24.

[29] R. Rocha-Chávez, M. Shapiro, F. Sommen, Integral theorems for functions and differential forms in $\mathbb{C}^{m}$, Research Notes in Mathematics 428, Chapman \& Hall/CRC, Boca Raton, FL, 2002.

[30] J. Ryan, Complexified Clifford analysis, Comp. Var. Theory Applic. 1(1) (1982/83), 119-149.

[31] I. Sabadini, F. Sommen, Hermitian Clifford analysis and resolutions, Math. Methods Appl. Sci. 25(16-18) (2002), 1395-1413.

[32] E.M. Stein, Singular Integrals and Differentiability Properties of Functions, Princeton Math. Ser. 30, Princeton Univ. Press, Princeton, 1970. 